\title{
El trabajo con neologismos en la enseñanza del español: el lenguaje de los platos gastronómicos ${ }^{1}$
}

\section{O Trabalho com neologismos no ensino de espanhol: a linguagem dos pratos gastronômicos}

\author{
Working with neologisms in Spanish teaching: the language \\ of gastronomic dishes
}

Glauber Lima Moreira²

Lucimara Alves Costa ${ }^{3}$

Fabiana da Silva França4

\begin{abstract}
RESUMEN: Las investigaciones sobre lenguas extranjeras (LE) han logrado considerables avances en los últimos años, principalmente dentro del campo de los estudios acerca de las metodologías adecuadas para el desarrollo de las clases de le para estudiantes extranjeros. Sin embargo, dichos estudiantes todavía afrontan varias dificultades encontradas durante el proceso de enseñanza-aprendizaje de LE, como el aprendizaje del vocabulario básico de la lengua extranjera. Un ejemplo son las palabras relacionadas con la gastronomía, que suponen un gran reto para el aprendiente no nativo, pues en este campo lexical se encuentra un abanico de nombres de platos que adquieren un nuevo significado para el significante $y$, por consiguiente, la comprensión del vocablo queda comprometida cuando se trata de un neologismo, es decir, denominaciones distintas para un concepto ya conocido, ya que
\end{abstract}

\footnotetext{
1 Trabajo elaborado a partir de las discusiones en la asignatura 'Terminología y Neología', ministrada por la profesora Dra. Teresa Cabré, en el Doctorado en Traducción y Ciencias del Lenguaje de la Universitat Pompeu Fabra (UPF).
}

2 Professor de Espanhol do Curso de Bacharelado em Turismo da Universidade Federal do Piauí (UFPI). Graduado em Letras com habilitação em Português e Espanhol pela Universidade Estadual do Ceará (UECE) e mestre em Linguística Aplicada pela mesma instituição. Doutorando em Traducción y Ciencias del Lenguaje na Universitat Pompeu Fabra (UPF) com bolsa CAPES. É membro dos Grupos de Pesquisa: Grupo de Estudos e Pesquisas Interdisciplinar em Turismo (EITUR/UFPI); Lexicologia, Terminologia e Ensino (LETENS/UECE), e do Grupo INFOLEX/IULA, da Universitat Pompeu Fabra (UPF), Espanha.

${ }^{3}$ Professsora de Português para Estrangeiros na Pontificia Universidad Católica de Valparaíso (PUCV), Chile. Doutora em Linguística pela la Universidade Estadual Paulista (UNPES/IBILCEBrasil) e pela Universitat Pompeu Fabra (UPF). E-mail: lucimara.costa@hotmail.com

${ }^{4}$ Doutora pela Universidade Federal da Paraíba com período de pesquisa na Universitat Pompeu Fabra (UPF) com bolsa CAPES. Bibliotecária da Universidade Federal de Campina Grande. Email: fabiana21franca@gmail.com 
El trabajo con neologismos en la enseñanza del español:

el lenguaje de los platos gastronómicos

existe una denominación anterior con una motivación primaria (SAGER, 1993). Así, nuestro objetivo es analizar los neologismos encontrados en menús gastronómicos de restaurantes y comprobar si están contemplados en los diccionarios analizados y cómo están clasificados. Por tanto, en este estudio, comprobamos, a través de algunos artículos lexicográficos, que dichos repertorios no ofrecen a sus consultantes, los neologismos existentes.

PALABRAS CLAVE: Neologismo; Léxico; Gastronomía; Diccionario; Aprendizaje de español.

RESUMO: As investigações sobre línguas estrangeiras (LE) têm alcançado consideráveis avanços nos últimos anos, principalmente dentro do campo dos estudos sobre as metodologias adequadas para o desenvolvimento das aulas de língua espanhola para estudantes estrangeiros. No entanto, estes estudantes ainda enfrentam várias dificuldades durante o processo de ensino-aprendizagem de uma LE, inclusive na aprendizagem do vocabulário básico da língua estrangeira. Um exemplo disso são as palavras relacionadas à gastronomia, que representam um significativo desafio para o aprendiz não nativo, pois neste campo lexical se encontra uma grande variedade de nomes de pratos que, muitas vezes, se tratam de neologismos, isto é, denominações distintas para um conceito já conhecido, uma vez que já existe uma denominação anterior com uma motivação primária (SAGER, 1993). Nesse sentido, nosso objetivo é analisar os neologismos encontrados em menus gastronômicos de restaurantes e comprovar se estão contemplados nos dicionários analisados y como estão classificados. Portanto, neste estudo, comprovamos, através de alguns verbetes, que tais dicionários não oferecem aos seus usuários os neologismos existentes.

PALAVRAS-CHAVE: Neologismo, Léxico, Gastronomia, Dicionário, Ensino de espanhol.

ABSTRACT: Investigations on foreign languages (FL) have reached considerable advances within the past few years, mainly in the research field of methodologies directed to the development of lectures in spanish language to foreign students. However, these students still face several difficulties during the process of teachinglearning a foreign language, including learning basic vocabulary of the foreign language. One example of this are the words related to gastronomy which represent a significant chalenge to the non-native learner, because within this lexical field, there is a wide variety of dishes names that, many times, are secondary neologisms, which means, there are distinct denominations to a known concept, once there is already a former denomination with a primary motivation (SAGER, 1993). Therefore, our aim is to analyze the neologisms found in gastronomic menus of spanish restaurants and how they are categorized. Therefore, in this study, it is proven, through some entries, that dictionaries do not provide existing neologisms to their users.

KEYWORDS: Neologism; Lexicon; Gastronomy; Dictionary, Spanish language learning.

\section{Introducción}


El estudio de las unidades neológicas de una lengua (materna o extranjera) permite dar cuenta de la vitalidad de la misma y del grado de productividad de los procesos morfosintácticos y semánticos utilizados en un código lingüístico dado. En este sentido, el presente trabajo persigue el objetivo de analizar los neologismos presentados en menús gastronómicos de restaurantes españoles y su clasificación. Para ello, partimos de un análisis de diccionarios generales de lengua española -Diccionario de Español para Extranjeros (SM, 2002), Diccionario de la Lengua Española (en línea DLE, 2015) y Plats a la carta (TERMCAT, 2017)- para verificar si los términos seleccionados en estos menús están registrados o no, es decir, si estamos delante de un caso de neonimia ${ }^{5}$ o si son términos que ya pasaron por el proceso de desterminologización y actualmente forman parte del léxico común y, por eso, se encuentran registrados en dichas obras. Asimismo, nuestro propósito es verificar cómo el diccionario, así como otras fuentes de investigación, como, por ejemplo, los bancos de datos neológicos, pueden aprovechar el diccionario como herramienta didáctica en el aula para trabajar con el contexto de los neologismos en la enseñanza del español como lengua extranjera (en adelante ELE).

Nuestro interés en realizar esta investigación se debió, primordialmente, a la pretensión de discutir sobre la neología y su enseñanza en las clases de ELE, y examinar la posible contribución del repertorio lexicográfico en lo relativo a cubrir las necesidades de comprensión (en los menús) de dichas palabras desconocidas por el consultante extranjero de ELE.

\section{Neología: panorama general}

En líneas generales, la neología puede definirse como una disciplina que se ocupa de las "novedades" o unidades y conceptos nuevos que surgen y se incorporan a una lengua, es decir, el estudio de los neologismos. Como señaló

\footnotetext{
${ }^{5}$ neonimia: neologismos empleados dentro de un determinado ámbito de especialidad.
} 
acertadamente Cabré (2011), el término Neologie apareció por primera vez en francés. De acuerdo con los estudios de J. C. Boulanger (2010), en el año 1758, el referido vocablo apareció con el sentido de "creación de palabras, expresiones o sentidos nuevos"; sin embargo, con el tiempo, este mismo término ha llegado a adquirir un sentido de transgresión de la norma, de tal manera que, en los siglos XVIII y XIX, el vocablo neologismo comenzó a ser utilizado como "creación abusiva de términos nuevos" (Cabré, 2011, p. 3) y, por consiguiente, causó un cierto desinterés por parte de la lingüística en desarrollar estudios científicos referentes a este tema.

Durante los años 70, es importante decir que, por influencia de la terminología, se percibe y comienza un mayor interés por las investigaciones acerca de los neologismos. De ahí, siguiendo esta reciente perspectiva científica y con base en los supuestos de Boulanger (1988), el término neología adquiere nuevos significados para referirse a:

- El proceso de creación de unidades léxicas a través de los recursos de la propia lengua o de recursos externos a ella;

- El estudio teórico y aplicado de innovaciones léxicas;

- La actividad institucional destinada a crear, recopilar, consignar, difundir e implantar unidades léxicas nuevas;

- La actividad de identificación de ámbitos especializados emergentes que requieren terminología;

- La relación con los diccionarios (CABRÉ, 2011, p. 4).

Según el Diccionario de la Lengua Catalana, el neologismo "[...] es una unidad léxica nueva, formal o semánticamente, creada en una lengua por las propias reglas de formación de palabras o prestada a otra lengua ${ }^{6}$." En este sentido, Sager (1993, p. 125) señala que el surgimiento de neologismos en el área de la ciencia y tecnología es el resultado de la necesidad de encontrar una denominación única para los nuevos conceptos. Por lo que respecta a la forma, tenemos básicamente dos tipos de neologismos: creaciones totalmente nuevas

${ }^{6}[\ldots]$ és una unitat léxica nova, formalment o semànticament, creada en una llengua per les pròpries regles de formació de mots o manllevada a una altra llengua. 
o préstamos de otras lenguas y, por consiguiente, dos situaciones de creación de neologismos (primaria y secundaria), que pueden ser resumidas de la siguiente forma:

\section{Cuadro 1: Creación de neologismos}

\begin{tabular}{|l|l|}
\hline Formación primaria de términos & Formación secundaria de términos \\
\hline $\begin{array}{l}\text { - es el resultado de innovación científica y y } \\
\text { tecnológica }\end{array}$ & $\begin{array}{l}\text { - es el resultado de la transferencia de } \\
\text { conocimiento de una comunidad a otra }\end{array}$ \\
\hline - siempre acompaña la fomación del concepto & $\begin{array}{l}\text { - crea una denominación nueva para un } \\
\text { concepto ya existente }\end{array}$ \\
\hline $\begin{array}{l}\text { - ocurre dentro de una misma lengua } \\
\text { (monolingüe) }\end{array}$ & ocurre entre lenguas distintas (multilingue) \\
\hline - es más o menos espontánea & - puede ser planeada o diseñada \\
\hline $\begin{array}{l}\text { - puede ser provisoria o solidificarse (tornarse } \\
\text { definitiva) }\end{array}$ & $\begin{array}{l}\text { - puede ser provisoria o solidificarse (tornarse } \\
\text { definitiva) }\end{array}$ \\
\hline $\begin{array}{l}\text { - no existe una denominación previa (creación } \\
\text { ex nihilo) }\end{array}$ & $\begin{array}{l}\text { - ya existe una denominación anterior con una } \\
\text { motivación primaria }\end{array}$ \\
\hline - es creada por expertos & $\begin{array}{l}\text { - es creada por terminólogos y traductores } \\
\text { (por no encontrar un equivalente exacto o } \\
\text { satisfactorio en la lengua meta) }\end{array}$ \\
\hline
\end{tabular}

Fuente: Adaptado de Sager (1993)

De acuerdo con el autor mencionado antes, existen diversos aspectos sociales que impulsan el surgimiento o la creación de neologismos, citamos los tres que consideramos principales: (i) la adopción del inglés como lengua oficial del ámbito científico, lo que justifica que la mayor parte de neologismos de creación primaria se produce en inglés, así como el mayor número de préstamos también proviene de la lengua inglesa; (ii) la diferencia en el desarrollo científico-tecnológico entre los países, lo que hace que haya un desequilibrio en el desarrollo de la terminología entre distintos países, por ejemplo, entre Estados Unidos y Canadá y países de América Latina, o incluso entre otros países europeos; (iii) distintos recursos para la formación de términos y distintas necesidades, lo que motiva la variación terminológica.

Sin embargo, como señala Cabré et al. (2002), una de las grandes dificultades en tratar los neologismos es exactamente la relatividad de su 
concepto, su definición y delimitación, puesto que resulta muy difícil delimitar con parámetros objetivos y cerrados el significado del término neologismo,

El neologismo es un elemento de gran complejidad. Por un lado ha sido considerado, por su vaguedad, un concepto inestable y relativo; por otro, presenta tantas vertientes de análisis que parece imposible dar cuenta de todas ellas al mismo tiempo; y, finalmente, escapa a las posibilidades de la lingüística de tratarlo exclusivamente como un objeto propio de su campo de estudio. Intentar, pues, dar cuenta de un objeto multidimensional y multidisciplinario no es una tarea fácil de abordar por parte de los lingüistas. (CABRÉ, 20011, p. 01)

De hecho, ellos mismos tratan de establecer parámetros que puedan detectar e identificar una nueva palabra. Rey (1976) establece tres parámetros de diferente naturaleza a través de los cuales es posible establecer conjuntos de neologismos:

a) criterio temporal: considera neologismo cualquier palabra que haya surgido en un período reciente;

b) criterio psicolingüístico: un neologismo será aquella unidad léxica que los hablantes identifican como nueva;

c) criterio lexicográfico: considera como neologismo una palabra que no está documentada en un determinado corpus lexicográfico.

Sin embargo, como pone de relieve Estopà (2009, p. 41), tanto el criterio temporal como el psicolingüístico son bastante subjetivos $y$, por tanto, menos eficientes cuando se quiere realizar un trabajo en equipo, como el realizado por los miembros del Observatorio de Neología (OBNEO) de la Universitat Pompeu Fabra (UPF), razón por la cual se considera el criterio lexicográfico el camino más adecuado y eficiente para asegurar la sistematicidad de los datos obtenidos.

Por tanto, la neología, en general, se clasifica en dos tipos distintos, a saber: a) la neología nominativa, que tiene por objeto designar nuevas realidades (objetos y conceptos) y b) la neología estilística, que se corresponde con la búsqueda de una mayor expresividad del discurso. Esta última, a 
menudo, se caracteriza por su fugacidad y es observada principalmente en la literatura y en el lenguaje/discurso humorístico.

En este sentido, Correia y Almeida (2012) explican que, además de estos dos tipos, está también la neología de la lengua que está formada por unidades léxicas del habla que, al no distinguirse de las otras, no despiertan ningún sentido de novedad en el hablante. Como ejemplos de ello podemos citar las formaciones compuestas de afijos, es decir, las que van pospuestas a la base léxica a través de la terminación -mente (seguramente, reconocidamente, etc.).

Las referidas autoras señalan que, por un lado, lo que hace que estas unidades sean consideradas neológicas es que no están contempladas en el diccionario; por otro lado, todavía se discute la importancia y necesidad de su inclusión en los repertorios lexicográficos, pues si tenemos en cuenta, en términos de complementariedad entre el diccionario y la gramática, que el repertorio lexicográfico debe contener solamente las características idiosincrásicas de las unidades léxicas, las unidades resultantes neología de la lengua, por no tener estas características, no tendrían que estar registradas en dichos libros (Correia y Almeida, 2012, p. 19). Por tanto, la expansión del léxico de un código lingüístico puede pasar a través de procesos derivados de la lengua materna o préstamos de otros sistemas lingüísticos.

Para ello, según Correia y Almeida (2012), la innovación léxica puede ocurrir por medio de tres mecanismos lingüísticos:

a) Neología formal: en ella, las palabras creadas provienen de procesos propios de la lengua a partir de la combinación de morfemas, sean bases 0 afijos.

b) Neología semántica: consiste en el uso de las unidades léxicas existentes con nuevos significados.

c) Neología por préstamo: se trata de la importación de las unidades léxicas de otros sistemas lingüísticos, que pueden ser adaptadas, o no necesariamente, al nuevo idioma. 
El trabajo con neologismos en la enseñanza del español:

el lenguaje de los platos gastronómicos

Dicha clasificación, anteriormente presentada por Cabré (2006, p. 231-

234), puede ser, de hecho, pormenorizada de la siguiente manera:

\section{Neologismos de forma (formais)}

- Sufijación (FSUF): formado a partir de la adición de un sufijo a un radical (inserción, clonaje, empleabilidad).

- Prefijación (FPRE): formado por la adición de un prefijo a un radical (antitécnico, pregado).

- Interferencias entre sufijación y prefijación (FPRSU): casos en que los neologismos pueden clasificarse tanto como formados por prefijación como formados por sufijación, por lo que no puede dilucidarse cuál de los dos procesos es el último que se ha realizado (remasterización).

- Composición o composición patrimonial (FCOM): neologismo formado a partir de dos radicales (simples o complejos) (apoyabrazos, quitahambre).

- Composición culta (FCULT): que puede ser formada mediante tres procesos:

- una forma prefijada culta y una forma sufijada culta (biogenia, biodiversidad).

- una forma prefijada culta y un radical (autoexigencia, fotoperiodismo).

- un radical (propio de la lengua o bien prestado de otra lengua) y una forma sufijada culta (clasicómano, simpaticoide).

- lexicalización (FLEX): formado a partir de la lexicalización de una forma flexiva (no de un paradigma completo) normalmente de una forma del paradigma verbal (removida, encuadernado).

- conversión sintáctica (FCONV): formado a partir de un cambio de categoría gramatical sin modificación de la base léxica (neoliberal, clonar).

- sintagmación (FSINT): formado por una estructura sintáctica lexicalizada (trabajadora social, golpe de revés liftado).

- siglación (FTSIG): formado por las letras correspondientes a una sigla (ADN, $A R N, C P U)$.

- acronimia (FTACR): formado por la combinación de segmentos de palabras que forman una estructura sintagmática (turismática: informática aplicada al turismo).

- abreviación (FTABR): formados por abreviación de la base léxica de una unidad (prota (protagonista), neocon (neoconservador).

- por variación (FVAR): variante formal ortográfica (ni morfológica ni sintáctica) de una palabra documentada en el corpus de exclusión. Por ejemplo, infrastructura (infraestructura).

\section{Neologismos sintácticos (SINT)}

- Implican un cambio de subcategoría gramatical (género, número, cambio de régimen verbal, etc.) en una base léxica (amo de casa; ama de casa).

\section{Neologismos semánticos (S)}

- Formados por una modificación del significado de una base léxica (buscador (informática), llegador (jugador de fútbol). También puede tratarse de un 
El trabajo con neologismos en la enseñanza del español:

el lenguaje de los platos gastronómicos

neologismo formado a partir de un nombre propio (de persona o marca registrada) utilizado como nombre común (nivea, tupperware).

\section{Préstamos (M/AM)}

- Unidades importadas de otra lengua (búnker, sushi). En este caso pueden ser préstamos no adaptados (M) o préstamos adaptados (AM).

Sobre los dos últimos neologismos presentados, en especial para los casos de neonimia, conviene señalar las siguientes observaciones:

1- Con respecto a los neologismos semánticos, estos procesos pueden ocurrir por (1) ampliación de sentido, que puede acontecer por medio de (i) cambio del ámbito temático (virus (informática y medicina), rehabilitación (urbanismo y medicina) o (ii) desterminologización (chip, televisor, Alzheimer), esto es, surgió primeramente dentro de un ámbito especializado y después pasó a ser utilizado en la lengua general. También por (2) reducción de sentido, es decir, cuando los neologismos surgieron como parte de la lengua general, pasaron por un proceso de terminologización y se convirtieron en parte del léxico especializado. Ej.: ratón (informática). Y por (3) cambio semántico que puede ocurrir por el empleo de una metaforización o resemantización. Ej.: colonia de células, parche'.

2- La adopción de préstamos de otras lenguas, sean no adaptados como, por ejemplo, en "timing, baguette, lied, atrezzo" o préstamos adaptados o traducidos, como es el caso de los calcos, posean consecuencias gramaticales y políticas para el país que los utilice.

\footnotetext{
7 Esta palabra, que en el DLE (2001) alude a lo que todos conocemos como el pedazo de tela, papel, piel, etc., que se pega sobre una cosa, ha ampliado de manera sorprendente su significado, pues en el parlache pasó a definir el lugar de reunión de los jóvenes, posteriormente como sinónimo de fiesta o rumba, "qué parche tan play" (qué fiesta tan buena) y a formar locuciones verbales como "coger de parche" (burlarse reiteradamente de una persona) y "abrirse del parche" (irse del lugar). http://revistavirtual.ucn.edu.co/index.php/RevistaUCN/article/viewFile/135/260
} 
De hecho, tenemos países que prefieren utilizar el préstamo tal cual es en la lengua extranjera, principalmente en términos provenientes del inglés, como mouse, software, etc., como es el caso de Brasil y Chile, entre otros países. Y existen aquellos que optan por una adaptación de estos préstamos a la lengua de llegada (adaptaciones gráficas y fonéticas o morfológicas) o incluso una traducción de los mismos (calco léxico y sintáctico) como forma de mayor conservación de la propia lengua, como es el caso de España, por ejemplo, al presentar los términos ratón (mouse), diapositiva (slide) y programario o programa informático (software), en vez de utilizar los préstamos en inglés.

Por tanto, cabe decir que esta fue la clasificación que hemos considerado para el análisis de los datos del presente estudio, como presentamos a continuación.

\section{Enseñanza y aprendizaje de la neología en las clases de LE}

Al reflexionar sobre la creación neológica en una lengua, se debe considerar que ese factor está directamente relacionado con la cultura de estos pueblos, pues cada lengua refleja el punto de vista particular que tienen sus hablantes de observar la realidad y de conceptualizarla dentro de su cultura. De ahí, como ya comentara Diki Kidiri (2009) -terminólogo que participó activamente en la creación de terminología en lenguas africanas, en la época de la descolonización del continente -, cuando se denomina una nueva realidad en una lengua, sus hablantes seleccionan aquellos rasgos del objeto que tienen relevancia en su cultura.

En este sentido, como señala Zavaglia (2009, p. 08) "[...] una lengua puede potencialmente expresar cualquier contenido en función de su tradición 
histórica y cultural en términos de creación de vocabulario."8 Por tanto, según la misma autora "[...] el léxico tiene sus raíces en las costumbres, en las tradiciones, en la moral de una cultura particular y de sus habitantes; éste expresa una visión del mundo particular de una lengua específica, ${ }^{\prime \prime 9}$ es decir, el léxico refleja la realidad de la comunidad en la que el sujeto está incluido y, además de ser una herramienta necesaria para el proceso de comunicación, el léxico es, sobre todo, la representación de estas personas, de sus experiencias y de la historia vital de cada uno.

Así, como destacó Biderman (2001), aunque el léxico es patrimonio de la comunidad lingüística, en la práctica son los usuarios de la lengua los que crean, mantienen y cambian el vocabulario de dicho código, al atribuir connotaciones particulares a los lexemas, en la utilización del discurso, cambiando, en algunos casos, las áreas de significado de las palabras (Biderman, 2001, p. 179); por eso, la necesidad de que el léxico, en la escuela y otras instituciones educativas, pase a ser objeto de análisis y enseñanza en el estudio tanto de la lengua materna (en adelante LM) como en el de la lengua extranjera (en adelante LE), pues como bien destaca Andrade (2014):

\begin{abstract}
Al percibir la escuela como el espacio responsable de auxiliar al desarrollo intelectual y social de los miembros de estas comunidades, que están cambiando y desarrollándose en el tiempo, se convierten en pertinentes investigaciones dirigidas a la enseñanza del léxico en el aula, por ejemplo, investigar las causas que generan la creación de nuevas unidades lexicales en el campo de una lengua. ${ }^{10}$ (ANDRADE, 2014, p. 58)
\end{abstract}

\footnotetext{
8 [...] uma língua pode, potencialmente expressar qualquer conteúdo, dependendo de sua tradição histórico-cultural em termos de criação vocabular.

${ }^{9}$ [...] o léxico é enraizado aos costumes, às tradições, à moral de uma determinada cultura e de seus habitantes; ele expressa uma visão de mundo particular de uma língua específica.

${ }^{10}$ Ao enxergar a escola como o espaço responsável por auxiliar o desenvolvimento intelectual e social dos membros dessas comunidades, que vão mudando e se desenvolvendo ao longo dos tempos, tornam-se relevantes pesquisas voltadas para o ensino do léxico na sala de aula, como, por exemplo, investigar os motivos que geram a criação de novos itens lexicais dentro de uma língua.
} 
Sin embargo, a pesar de que se destaque la importancia de la realización de investigaciones dirigidas a la enseñanza del léxico en clase, sobre todo al trabajo con los neologismos, se confirma que, en el caso de este trabajo, el idioma castellano, estableciendo una comparación con la enseñanza de la neología en las clases de lengua portuguesa (contexto brasileño), observamos que en la sección dedicada al léxico, los Parámetros Curriculares Nacionales (PCNs), documentos oficiales del Ministerio de Educación en los cuales se dan sugerencias para guiar al docentes (y también a los discentes) en la construcción de las relaciones lexicales con el fin de ampliar su repertorio lexical, no hacen ninguna mención explícita a la relevancia de trabajar con neologismos en el ámbito educacional.

De hecho, cuando en la sección de Práctica de Análisis Lingüístico entendemos que hablan sobre el aprendizaje de nuevas palabras, se refieren a las palabras que no forman parte del conjunto del vocabulario del aprendiz y no explícitamente a la neología, como se puede observar a continuación:

Ampliación del repertorio léxico mediante la enseñanza y el aprendizaje de nuevas palabras, con el fin de permitir: [...] el empleo adecuado de palabras limitadas a ciertas condiciones históricas y sociales (regionalismos, extranjerismos, arcaísmos, neologismos, jerga, argot). ${ }^{11}$ (BRASIL, 1998, p. 62$63)$.

Como se pode constatar, como señala Resende (2014, p. 56), decir que los neologismos son palabras establecidas siguiendo ciertas condiciones lingüísticas es, en este sentido, una afirmación dudosa, puesto que no se contempla que una de las razones necesarias para crear nuevas palabras es la de nombrar las "realidades nuevas".

En definitiva, podemos concluir que, al emplear el neologismo en una perspectiva muy amplia, los PCNs no consideran importante el papel que

\footnotetext{
${ }^{11}$ Ampliação do repertório lexical pelo ensino-aprendizagem de novas palavras, de modo a permitir: [...] o emprego adequado de palavras limitadas a certas condições histórico-sociais (regionalismos, estrangeirismos, arcaísmos, neologismos, jargões, gíria).
} 
aquellos puedan desempeñar en las prácticas y estrategias de lectura (y en la producción textual), ya que el mismo razonamiento que es utilizado para entender y construir el significado de un neologismo puede ser usado, a menudo, para comprender el significado y el uso de una unidad lexical, ya sea un neologismo o no.

\section{¿EI diccionario podría configurarse como herramienta didáctica para trabajar con neologismos en las clases de LE?}

Como ha señalado Carvalho $(1987$, p. 08$)$ y varios lingüistas, entre ellos (Leffa, 2011; Biderman 2002), la relación intrínseca entre el léxico y la sociedad. En este sentido, los neologismos o el proceso de creación lexical deben ser considerados teniendo en cuenta el periodo temporal y la comunidad lingüística, así como la experiencia y la historia de esa comunidad.

Junto a esta relación encontramos el diccionario, que, según Hartmann (1983, p. 03-04), es un "[...] libro de referencia o lista de palabras (por lo general en orden alfabético), que aportan informaciones sobre estas unidades lexicales como, por ejemplo, el significado, la pronunciación $y$, en algunos casos, el equivalente en otras lenguas." ${ }^{\prime 12}$

Sin embargo, como ha señalado Costa $(2015$, p. 36), el diccionario es mucho más que un simple libro de referencia y una herramienta lingüística utilizada para contestar a las cuestiones concretas y específicas de sus consultantes, el diccionario es, ante todo, una herramienta social, pues describe el léxico de un idioma $y$, al mismo tiempo, transmite las convicciones, las creencias y las ideologías de un pueblo, mediante la selección del léxico registrado en su nomenclatura. Pero, ¿qué sucede con los neologismos? ¿Deberían estar registrados en el diccionario? ¿Cómo podría funcionar el diccionario como herramienta para el trabajo con neologismos en el aula?

\footnotetext{
12 [...] livro de referência ou lista de palavras (normalmente em ordem alfabética), que trazem consigo informações sobre estas unidades léxicas, tais como: significado, pronúncia e, em alguns casos, equivalentes em outras línguas.
} 
Como ya hemos mencionado antes, una de las características que nos permite identificar un neologismo es justamente el hecho de que este no está todavía incorporado en la obra lexicográfica, como afirma Cabré et al. (2002):

En el Observatorio de Neología del Institut Universitari de Lingüística Aplicada (IULA) de la Universitat Pompeu Fabra (UPF), "[...] se considera neologismo cualquier palabra que no aparece en un corpus lexicográfico de exclusión previamente establecido, formado por diccionarios prescriptivos y por diccionarios descriptivos ${ }^{13 \prime \prime}$. (CABRÉ et al., 2002, p. 288-289).

Por ello, el diccionario sería el último lugar en el que un neologismo debería ser registrado, una vez que eso ocurra ya no estaremos delante de un caso de neología. En este sentido, el diccionario puede funcionar perfectamente como una herramienta para la enseñanza de neologismos en el contexto escolar, siendo el lugar de referencia para constatar si la unidad léxica a ser analizada es o no neológica, lo que consiste en adoptar el criterio lexicográfico apuntado por Rey (1976) y considerado como el más adecuado y sistemático por el OBNEO de la UPF.

Un segundo paso sería considerar el criterio psicolingüístico, relacionado con el conocimiento del mundo del hablante y las informaciones disponibles por el diccionario. En otras palabras, verificar si los estudiantes identifican esa palabra como nueva o si les parece conocida, lo que puede conducirnos a dos tipos de situaciones:

1. Si ya han tenido contacto con ese vocablo antes, averiguar: ¿Dónde y cómo ocurrió? ¿Ya conocían ese concepto antes? ¿Y esa denominación? ¿EI concepto se parece a alguna otra palabra recogida en el diccionario?

2. Si la identifican como una palabra totalmente nueva, ¿cuál es su significado? ¿Existe alguna semejanza entre ese concepto y el concepto

13 [...] es considera neologisme qualsevol paraula que no apareix en un corpus lexicográfic d`exclusió prèviament establert, format per diccionaris prescriptius i per diccionaris descriptius. 
atribuido a alguna otra unidad registrada en el diccionario? ¿Qué la justifica? ¿Cuál es la necesidad de crear ese neologismo?

$\mathrm{Si}$ estamos ante la primera situación podremos tener distintas posibilidades: (i) puede tratarse de un neologismo no demasiado reciente, surgido hace algún tiempo, pero no muy conocido y propagado por los medios de comunicación, lo que hace que algunos lo conozcan y otros no; (ii) puede tratarse de un mismo concepto conocido con una denominación distinta, es decir, un caso de variación terminológica y, por tanto, puede que exista alguna lexía diccionarizada como un concepto similar al presentado en esta nueva unidad.

En cuanto a la segunda situación, cuando se trata de una palabra reconocida como totalmente nueva, la primera decisión que se tiene que realizar es recuperar su significado. Para ello, debemos buscar los medios de divulgación de ese neologismo, en nuestro caso, los menús de restaurante y, en función de ello, relacionar el conocimiento del mundo de los estudiantes y de las informaciones de los diccionarios como medio de referencia. Por ejemplo, como se trata de estudiantes con lengua materna distinta del español, hay que observar si logran relacionar el concepto representado por esa unidad con alguna otra unidad en su propia lengua, es decir, el equivalente en su lengua materna. El diccionario puede facilitar este entendimiento, pues, aunque no contenga el neologismo en cuestión, puede registrar las otras unidades reconocidas como similares por los estudiantes.

Esa situación nos permitirá pensar en el porqué de crear esa nueva palabra, su contexto y necesidad de creación. Si se trata de una creación ex nihilo, es decir, creada de cero, a partir de ninguna otra existente, podemos 
pensar en el contexto y el motivo de ser creada, como es el caso, por ejemplo, del neologismo "meme"14.

En la mayoría de las veces estos neologismos surgen por una necesidad de acompañar el desarrollo tecnológico, como para denominar un nuevo producto que está saliendo en el mercado, por la propagación mediática y surgimiento de un nuevo fenómeno que no tenga una denominación anterior o incluso, cuando se trata de neologismos secundarios, es decir, que ya posean una denominación o motivación primaria en otra lengua. Muchas veces ellos ocurren en el proceso de traducción de una lengua a la otra, pues por no haber equivalentes considerados satisfactorios en la lengua de llegada, el traductor se ve frente a dos opciones más frecuentes: conservar el préstamo o presentar un nuevo término en la lengua meta, y para que se cree ese nuevo término el punto de partida es buscar equivalentes en el diccionario.

Como se puede observar, el diccionario, desde que sea bien explotado, tanto por los profesores como por los aprendientes (cf. Moreira, 2013; 2017), puede sí configurarse en una herramienta para el trabajo con neologismos en el aula, incluso cuando se trata de los términos propios del ámbito de la gastronomía como vamos a presentar a continuación.

\section{Métodos y análisis del corpus}

Para la realización del presente estudio, realizamos la identificación y clasificación de los neologismos extraídos de algunos menús gastronómicos. En efecto, elegimos diez cartas de restaurantes de la ciudad de Barcelona

\footnotetext{
${ }^{14}$ El neologismo meme se escribe en redonda y sin comillas. Este término, creado por Richard Dawkins en su libro El gen egoísta, aparece definido en el diccionario Webster como 'idea, comportamiento, moda o uso que se extiende de persona a persona dentro de una cultura'. Por otra parte, meme se emplea cada vez más para referirse a cualquier imagen o texto, a menudo de contenido humorístico, que se comparte viralmente en las redes sociales durante un periodo de tiempo breve. Aunque no aparece recogido en los principales diccionarios de español, su grafía es respetuosa con las normas ortográficas y puede considerarse un término correcto, válido para ambas acepciones, y escribirse sin ningún resalte tipográfico.
} 
(España). Es importante señalar que su elección se hizo de manera aleatoria, disponible en las páginas de Internet.

El corpus tomado de dichas cartas gastronómicas contiene más de 564 nombres de platos. Para la identificación del corpus utilizado para el análisis de este estudio, procedimos a la extracción de los candidatos a neologismos. De ahí que, para el desarrollo de esta etapa, hayamos hecho uso del criterio lexicográfico, según el cual consideramos que una unidad es neológica si no aparece en los repertorios lexicográficos, ya sean generales o especializados, independiente de su tipología.

Para la clasificación de los neologismos, utilizamos la propuesta elaborada por Cabré (2006), expuesta anteriormente al tratar sobre los neologismos. Como una forma de recordar esa clasificación, la representamos con los códigos indicados a continuación:

1. Neologismos de forma (F)

- sufijación (FSUF)

- prefijación (FPRE)

- interferencias entre sufijación y prefijación (FPRSU)

- composición (FCOM)

- composición culta (FCULT)

- lexicalización (FLEX)

- conversión sintáctica (FCONV)

- sintagmación (FSINT)

- siglación (FTSIG)

- acronimia (FTACR)

- abreviación (FTABR)

- variación (FVAR)

2. Neologismos sintácticos (SINT)

3. Neologismos semánticos $(S)$

4. Préstamos (M/AM) (préstamo y préstamo adaptado, respectivamente)

5. Otros (casos extraños difíciles de etiquetar)

En este contexto, Cabré (2006, p. 241) afirma que "hay que recordar que los neologismos que no son préstamos, pero sí calcos de otras lenguas (traducciones literales de una palabra o sintagma), y se etiquetan según el proceso de formación correspondiente." De ahí, el uso del criterio lexicográfico, es decir, la presencia de una determinada unidad en un corpus de exclusión 
compuesto por diccionarios representativos de una lengua dada, es el método utilizado mayoritariamente para la identificación de los neologismos. Se trata de un criterio categórico, esto es, basta contrastar una lista con las palabras del corpus analizado con la lista de los temas de las obras lexicográficas que integran el corpus de exclusión.

Por tanto, para la realización del análisis de nuestro estudio, empleamos los siguientes corpus lexicográficos de exclusión:

a) Diccionario de Español para Extranjeros (SM, 2002)

b) Diccionario de la Lengua Española (en línea DLE, 2015)

c) Plats a la carta (TERMCAT, 2017)

Conviene señalar que hemos elegido como primer diccionario el "Diccionario de Español para Extranjeros" en su formato impreso, porque es una obra elaborada con fines específicos para la enseñanza de Español como lengua extranjera (ELE), o sea, dirigida a estudiantes no nativos. El motivo de la elección del DLE (formato en línea) se debe a que es una obra lexicográfica general de significativo prestigio elaborada por la Real Academia Española y, principalmente, por ser una obra de gran relevancia para los estudios del área. $Y$, por último, el Plats a la carta (TERMCAT) en formato en línea, por ser un diccionario especializado. Asimismo, es importante advertir que la última obra lexicográfica mencionada no ha sido elaborada con fines específicos de enseñanza de lenguas extranjeras. Dicho diccionario tiene como objetivo ofrecer al sector de restaurantes un marco conceptual para la elaboración de cartas y menús. Al final, esto nos permitirá realizar una comparación entre las obras generales y la especializada.

A continuación, presentamos los términos del vaciado del corpus que sirvieron para el desarrollo de nuestra investigación (Tabla 1):

Cuadro 2 - Términos encontrados 
El trabajo con neologismos en la enseñanza del español:

el lenguaje de los platos gastronómicos

\begin{tabular}{|c|l|l|}
\hline Ítem & Términos & Nombres del plato en la carta \\
\hline 1. & CANOLISÚ & Canolisú \\
\hline 2. & PIRIPI & Piripi \\
\hline 3. & TAP & Tap de Cadaqués \\
\hline 4. & BOMBA & Bomba \\
\hline 5. & LOCURA DE COLORES & Locura de colores \\
\hline 6. & TIMBAL & Timbal de Rossinyols de temporada con butifarra negra \\
\hline 7. & ROMESCADA & Romescada de Rape \\
\hline
\end{tabular}

Fuente: Datos de la investigación (2015).

Es importante resaltar que no hemos considerado los siguientes términos en este análisis:

a) platos con nombres de restaurantes

b) platos con nombres propios

c) platos con nombres en otras lenguas diferentes del castellano

A continuación, presentamos los neologismos encontrados en tales menús y contrastamos si estos están contemplados en los repertorios lexicográficos de referencia empleados en esta investigación (Tabla 2). Así, se configura como neologismo lo que no se recoge en dichos repertorios y se analizan en la siguiente tabla 2:

Cuadro 3 - Términos contemplados en los diccionarios

\begin{tabular}{|l|c|c|c|}
\hline Términos & $\begin{array}{c}\text { DICCIONARIO DE ESPAÑOL } \\
\text { PARA EXTRANJEROS (SM) }\end{array}$ & DRAE & $\begin{array}{c}\text { PLATS A LA CARTA } \\
\text { (TERMCAT) }\end{array}$ \\
\hline CANOLISÚ & no & no & no \\
\hline PIRIPI & sí & sí & no \\
\hline TAP & no & no & no \\
\hline BOMBA & no & sí & no \\
\hline $\begin{array}{l}\text { LOCURA DE } \\
\text { COLORES }\end{array}$ & no & no & \\
\hline
\end{tabular}


El trabajo con neologismos en la enseñanza del español:

el lenguaje de los platos gastronómicos

\begin{tabular}{|l|l|l|l|}
\hline TIMBAL & no & no & no \\
\hline ROMESCADA & no & no & no \\
\hline
\end{tabular}

Fuente: Datos de la investigación (2015).

En la aproximación de neologismos en un diccionario específico para la enseñanza de Español como lengua extranjera (SM) de uso, un diccionario normativo y general como es el caso del DLE y un diccionario específico del área de la gastronomía, también normativo, como es el Plats a la carta (TERMCAT).

Cuadro 4 - Análisis del corpus

\begin{tabular}{|c|c|c|c|}
\hline \multirow{2}{*}{ Términos } & \multicolumn{3}{|c|}{ REPERTORIOS LEXICOGRÁFICOS } \\
\hline & $\begin{array}{l}\text { DICCIONARIO } \\
\text { DE ESPAÑOL } \\
\text { PARA } \\
\text { EXTRANJEROS } \\
\text { (SM) }\end{array}$ & DRAE & Plats a la Carta (TERMCAT) \\
\hline \multicolumn{4}{|c|}{ CONCEPTOS } \\
\hline CANOLISÚ & - & - & - \\
\hline PIRIPI & $\begin{array}{c}\text { adj.inv. col. (ESTAR) } \\
\text { Ligeramente } \\
\text { borracho: aunque } \\
\text { solo se ha tomado } \\
\text { una cerveza ya está } \\
\text { piripi. }\end{array}$ & $\begin{array}{c}\text { adj. coloq. ebrio (\|l } \\
\text { embriagado por la } \\
\text { bebida). Está } \\
\text { piripi. }\end{array}$ & - \\
\hline TAP & - & - & $\begin{array}{l}\text { tap de Cadaqués y helado de } \\
\text { chocolate negro (pastelito de } \\
\text { bizcocho) }\end{array}$ \\
\hline BOMBA & - & $\begin{array}{l}\text { La que servía para } \\
\text { proveer de agua la } \\
\text { caldera de una } \\
\text { máquina de } \\
\text { vapor.(alimenticia) }\end{array}$ & - \\
\hline $\begin{array}{l}\text { LOCURA DE } \\
\text { COLORES }\end{array}$ & - & - & - \\
\hline
\end{tabular}




\begin{tabular}{|l|c|c|c|}
\hline TIMBAL & - & - & - \\
\hline ROMESCADA & - & - & - \\
\hline
\end{tabular}

Fuente: Datos de la investigación (2015).

En suma, observamos en la Tabla 3 que, en los repertorios analizados para esta investigación, no se contemplan los términos con el mismo significado que en las cartas de los restaurantes investigados.

Así, haciendo uso de la clasificación de neologismos postulada por Cabré (2006), los términos analizados en este trabajo son neologismos semánticos (S), neologismos de forma (F) y uno de ellos (Tap) que hemos clasificado bajo la etiqueta Otros, pues lo consideramos un ejemplo de neologismo difícil de ser catalogado. Veamos la clasificación en la Tabla 4:

Cuadro 5 - Clasificación de los neologismos

\begin{tabular}{|c|l|l|}
\hline Ítem & \multicolumn{1}{|c|}{ Términos } & \multicolumn{1}{c|}{ Clasificación } \\
\hline 1. & CANOLISÚ & $\begin{array}{l}\text { Neologismo formado por acronimia de } \\
\text { "cannolini y Tiramissú" (FTACR). }\end{array}$ \\
\hline 2. & PIRIPI & Neologismo semántico (S) \\
\hline 3. & TAP & Otros \\
\hline 4. & BOMBA & Neologismo semántico (S) \\
\hline 5. & LOCURA DE COLORES & Neologismo formado por sintagmación (FSINT) \\
\hline 6. & TIMBAL & Neologismo semántico (S) \\
\hline 7. & ROMESCADA & $\begin{array}{l}\text { Neologismo formado por sufijación (FSUF), } \\
\text { constituido por la adición del sufijo -ada al } \\
\text { radical romesc-o. }\end{array}$ \\
\hline
\end{tabular}

Fuente: Datos de la investigación (2015).

Por tanto, teníamos, en primer lugar, la intención de observar si con este análisis encontraríamos neologismos en los menús seleccionados para componer el corpus del presente estudio. En segundo lugar, pretendíamos averiguar $\mathrm{y}$, ulteriormente, comparar las definiciones de las palabras elegidas y 
extraídas de los menús (mencionados antes) en obras lexicográficas de referencia, es decir, el criterio aquí utilizado se llevó a cabo para determinar el carácter neológico o no de una unidad léxica, para determinar si en el vaciado existía alguna forma neológica.

\section{Consideraciones finales}

En síntesis, hemos podido observar que ciertos términos de una determinada área del conocimiento de una lengua no están todavía contemplados en los repertorios lexicográficos $y$, por esto, es necesario que los profesores conozcan bien tales vocablos para que puedan explicar y presentar a sus alumnos que están ante neologismos. En este sentido, estamos de acuerdo con que el uso adecuado del diccionario en el aula podrá presentar un carácter didáctico y contribuir eficazmente al proceso de enseñanza y aprendizaje de los neologismos $y$, consecuentemente, fomentar el conocimiento de ELE en el momento en que los aprendices confirman que determinadas unidades léxicas no están registradas en los repertorios lexicográficos, ya que son palabras nuevas.

Sabemos que la lengua es dinámica, está viva y llena de novedades. Por eso, todos los días surgen palabras nuevas, usos nuevos de palabras ya existentes, así como el desuso de algunos vocablos, es decir, lo que conocemos por arcaísmos. Por ello, las nuevas acuñaciones revelan un gran campo de investigaciones lingüísticas, pues se trata de creaciones neológicas generadas por la vitalidad de la lengua, la evolución científico-tecnológica y la interacción y desarrollo social, lo que produce la necesidad de crear nuevas palabras para representar nuevas realidades y acontecimientos de diferentes tipos $y$ contextos.

Por consiguiente, podemos concluir, de manera general que los datos de este estudio neológico nos revelan que existen términos relacionados con el área de la gastronomía para algunos de los cuales encontramos cambios de tipo semántico y formal, por ejemplo. En otras palabras, podemos identificar en el 
presente análisis la vitalidad de la lengua española por lo que respecta a la utilización de neologismos que se incorporan a los nombres de los platos de las comidas. De ahí, podemos afirmar que el neologismo presente en el contexto investigado puede convertirse, sobre todo para los aprendices extranjeros, en un tema difícil, e, incluso, en un obstáculo y en una falta de motivación para el desarrollo de la enseñanza y aprendizaje de ELE y, consecuentemente, un inconveniente en la comprensión eficiente del léxico de la lengua extranjera de estudio.

\section{Referencias}

ADRIÀ, Ferran. A muchos les parece que la creatividad es una palabra demasiado importante para la comida. Fietta Jarque, El País. Recuperado en 06.08.2005.

ALICIA, Fundación et al. Léxico científico gastronómico: las claves para entender la cocina de hoy. Barcelona: Planeta, S.A., 2006.

ALVES, Ieda Maria. Neologismo. Criação lexical. São Paulo: Ática, 1990.

ANDRADE, Adriano Bezerra. Neologia por empréstimo no livro didático de língua portuguesa: um estudo de caso. Dissertação de Mestrado. Universidade Federal de Tocantins (UFT). Araguaína, 2014.

BALDINGER, Kurt. Lengua y cultura: su relación en la lingüística histórica. Revista Española de Lingüística, 15, 02, 1985, pp. 247-276.

BARBOSA, Maria Aparecida. Léxico, produção e criatividade. 3. ed. São Paulo: Editora Plêiade, 1996.

BIDERMAN, Maria Teresa Camargo. A formação e a consolidação da norma lexical e lexicográfica no português do Brasil. En: NUNES, José Horta; PETTER, Margarida (Org.). História do saber lexical e constituição de um léxico brasileiro. São Paulo, SP: Humanitas, FFLCH-USP e Pontes, 2002.

BIDERMAN, Maria Teresa Camargo. Teoria Lingüística. São Paulo: Martins Fontes, 2001. 
BOCUSE, Paul. La cocina del mercado. Barcelona: Ediciones Destino. 2003.

BRASIL. Ministério da Educação e do Desporto. Parâmetros Curriculares Nacionais. $3^{\circ}$ e $4^{\circ}$ Ciclos do ensino fundamental - Língua Portuguesa. Brasília: MEC/SEF, 1998.

CABRÉ, María Teresa et al. La lexicografía i la identificació automatitzada de neologialèxica. Observatori de Neologia. Institut Universitari de Linguística Aplicada. Barcelona, 2002.

CABRÉ, María Teresa; ESTOPÀ BAGOT, R.; VARGAS SIERRA, Chelo. Neology in specialized communication. En: Terminology 18(1). Amsterdam: John Benjamins. ISSN 0929-9971, 2012, pp. 1-8.

CABRÉ, María Teresa; FREIXA, Judit; SOLĖ, Elisabet (ed.). Lèxic i neologia. Barcelona. Universitat Pompeu Fabra, 2002.

CABRÉ, María Teresa. Bases para una teoría de los neologismos léxicos: primeras reflexiones, 2011 [en prensa].

CABRÉ, María Teresa. La clasificación de neologismos: una tarea compleja. En: Alfa. São Paulo. Vol. 50, n², 2006, pp. 229-250

CABRÉ, María Teresa. La neología efímera. En: CABRÉ, María Teresa; FREIXA, Judit; SOLÉ, Elisabet (ed.) Lèxic i neologia [2a ed.]. Barcelona: Observatori de Neologia. Institut Universitari de Lingüística Aplicada. Universitat Pompeu Fabra; Documenta Universitaria. ISBN 978-84-96742-48-2, 2008, pp. 13-27.

CABRÉ, María Teresa. La terminología. Representación y comunicación. Barcelona: Institut Universitari de Lingüística Aplicada, 1999.

CABRÉ, María Teresa. Terminologie et linguistique: la théorie des portes. Terminologies nouvelles. Termologie et diversité culturelle, 21, 2000, pp. 10-15

CARVALHO, Nelly Medeiros de; OLIVEIRA, Rebeca Lins Simões de. Inovações lexicais na publicidade da mídia escrita e da mídia eletrônica - semelhanças e diferenças. En: ISQUERDO, Aparecida Negri; FINATTO, Maria José Bocorny (orgs). As ciências do léxico - lexicologia, lexicografia, terminologia. Vol. 4, Campo Grande: MS. UFMS, 2008.

CASSI, Davide; BOCCHIA, Ettore. La ciencia en los fogones: historia, técnicas y recetas de la cocina molecular italiana. Gijón: Trea, 2005. 
COSTA, Lucimara Alves da Conceição. Reflexões sobre a variação terminológica na Lexicografia corrente no Brasil e a construção das bases teóricometodológicas para o Dicionário de Lexicografia Brasileira. Tese de Doutorado. UNESP - São José do Rio Preto, 2015.

Diccionario de la lengua española. Madrid: Espasa, 2001.

Diccionario de nutrición y alimentos. Barcelona: Bellaterra, 1994.

Diccionario Plats a la Carta. Generalitat de Catalunya. Direcciò General de Política Lingüística. TERMCAT. Centro de Terminologia, 2010.

DOVAL, Gregorio. Diccionario de expresiones extranjeras. Madrid, Alianza editorial, 2004.

ESTOPÀ, Rosa. La neología especialitzada, repte constant per al mediador lingüístic. En: Coromina, Eusebi / Mestres, Josep M. [curadors] Aspectes de terminologia, neologia i traducció. Barcelona, Vic: Institut d'Estudis Catalans, Universitat de Vic. ISBN 978-84-9965-022-7, 2010, pp. 15-39.

ESTOPÁ, Rosa. Neologismes i filtres de neologicitat: aspectes metodològics. En: Cabré, María Teresa / Estopà, Rosa. (ed.). Les paraules noves: criteris per detectar i mensurar els neologismes. Vic, Barcelona: Eumo; Universitat Pompeu Fabra, 2009, pp. 41-48

GARCÍA PALACIOS, Joaquín. El trabajo sobre la neología terminológica en español. En: CABRÉ, María Teresa; DOMĖNECH, Ona; ESTOPÀ, Rosa; FREIXA, Judit; LORENTE, Mercè (eds.). Actes del I Congrés Internacional de Neologia de les Llengues Romàniques. Barcelona: Institut Universitari de Lingüística Aplicada. Universitat Pompeu Fabra; Documenta Universitaria. ISBN: 978-8492707-08-9, 2010.

GÓMEZ DE ENTERRÍA SÁNCHEZ, María Josefa; NAVARRO, Carmen. Creación neológica en vocabularios terminológicos en español pertenecientes a las áreas temáticas de Gestión del Turismo Gastronómico y Nueva Economía. Actes del I Congrés Internacional de Neologia de les Llengües Romàniques. Barcelona: IULA Institut Universitari de Lingüística Aplicada, Universitat Pompeu Fabra. 2010, p. 445-453.

GÓMEZ DE ENTERRIA, Josefa. El discurso especializado de la economía y el comercio. Una propuesta para la enseñanza en el aula de español de los negocios. En: CALVI, Maria Vittoria; SAN VICENTE, Félix (eds). Palabas de acá y de allá. La identidad del español y su didáctica. Lucca: Mauro Baroni editore. 
1999, pp. 61-78. Recuperado en

http://www.ub.edu/filhis/culturele/turismo.html

LEFFA, Vilson José. O dicionário deveria ser sistematicamente utilizado em aulas de línguas materna ou estrangeira como instrumento pedagógico? En: BEVILACQUA, Cleci Regina; HUMBLÉ, Philippe René Marie; XATARA, Claudia. (orgs.). Dicionário na Teoria e na prática - como e para quem são feitos. São Paulo, Parábola editora, 2011, pp. 123-132.

MARTÍN ZORRAQUINO, María Antonia. Formación de palabras y lenguaje técnico. En: Revista española de lingüística (REL), año 27, fasc. 2, juliodiciembre 1997, 1997, pp. 317-339.

MIRANDA, José Alberto. La formación de palabras en español. Salamanca. Ediciones Colegio de España, 1994.

MOREIRA, Glauber Lima; Pontes, Antonio Luciano. Diccionario: Un recurso didáctico. En: Moreira, Glauber Lima. et al. (org.). Reflexões e ações no ensino e aprendizagem de Espanhol/LE. Fortaleza: EdUECE, 2013.

MOREIRA, Glauber Lima. Dificultades de comprensión lectora en el artículo lexicográfico del léxico del turismo en los diccionarios SEÑAS y CLAVE. En: Revista Trama, V. 13, № 30, p. 249-271, 2017. Disponible en: http://erevista.unioeste.br/index.php/trama/article/view/15763/11863

MONTORO DEL ARCO, Esteban. Neología y creatividad lingüística. Valencia. Universidad de Valencia, 2012.

RESENDE, Priscila de. A neologia em sala de aula. Análise de atividades da coleção didática Português - uma proposta para o letramento. Priscila de Resende. Dissertação de Mestrado. Minas Gerais: Universidade Federal de Minas Gerais. Faculdade de Letras, 2014. 